\title{
Adaptive Optimization of Noisy Black-Box Functions Inherent In Microscopic Models
}

\author{
Eddie Davis, Aditya Bindal, and Marianthi Ierapetritou* \\ Department of Chemical and Biochemical Engineering \\ Rutgers - The State University of New Jersey \\ 98 Brett Road, Piscataway, NJ 08854, USA
}

\begin{abstract}
For stochastic systems where exact constitutive relations are unknown, a microscopic or molecular level description can be alternatively used. As microscopic simulations are computationally very expensive, there is a need for the development of robust algorithms capable of economically optimizing these noisy processes. In this paper, two approaches - an adaptive strategy using a gradient-based NLP method and a local response surface method - are applied to a stochastic reaction system. The effectiveness of these methods is evaluated in terms of the number of microscale function calls and computational time.
\end{abstract}

Keywords: Optimization, Noisy Functions, Microscopic Models, Black-Box

\section{Introduction}

Traditionally, system level optimization is performed on a macroscopic continuum level description of the physical system that presumes the accuracy of closed-form equations. However, for micro/nano applications and other systems where exact constitutive relations are unknown, a microscopic or molecular level description is the only realistic representation of the system physics. Furthermore, even though the questions of interest for system analysis are usually asked at the macroscopic level, recently researchers (Makeev et al., 2002) have shown that the details of the model description can have a profound effect on these analyses. Since closed-form equations are unavailable for the modelling and optimization at a fine resolution, coarse time-steppers can instead be used. Yet even though time-steppers provide a bridge between microscopic/stochastic simulations and computational optimization (Gear et al, 2002, Bindal et al., 2004), running microscopic simulations is computationally very expensive and therefore the use of standard optimization techniques can be prohibitive. Thus, there exists a need for the development of robust algorithms capable of economically optimizing these inherently noisy systems.

Different approaches have been employed in the past towards the optimization of noisy functions (Kelley, 1999). Global zero-order optimization methods such as genetic and evolutionary algorithms (Storn et al, 1995) can provide suboptimal solutions. Local zero-order methods utilizing function evaluations on sequences of simplices (Nelder-

\footnotetext{
*Author to whom correspondence should be addressed: marianth@soemail.rutgers.edu
} 
Mead, multidirectional search, and implicit filtering) yield optima with reasonable accuracy. However, no theoretical guarantee on convergence can be provided with the above methods. Although convergence bounds can be established with rigorous higherorder methods, the difficulty presented in using them lies in the fact that these algorithms can become trapped in artificial local optima introduced due to the presence of noise. To overcome this problem, an emerging strategy used is the response surface model (Jones et. al., 1998, 2001), in which the function is approximated by a low-order polynomial using the input-output response mapping, which is then used for optimization.

In this work we employ two iterative methods for the optimization of noisy functions arising from the description of systems at microscopic scales. We first develop a local optimization framework incorporating gradient-based methods coupled with an adaptive strategy to filter the objective function at different resolutions or scales, thereby providing bounds on convergence. Secondly, we employ a localized response surface method where part of the entire domain is approximated by a polynomial, which then traverses the region to identify local optima. The algorithmic features of the proposed methods are presented in the following sections.

\section{Proposed Approaches}

\subsection{Gradient-based Adaptive Method}

Consider a microscale model consisting of the macroscale inputs $x$ and the microscopic number of particles $N$ such that we are interested in optimizing an arbitrary objective function $g(x, N)$ over the parameter space of $x$. If the error in the function evaluation is assumed to be independent of $x$, then this function can be modelled as $g(x, N)=f(x)+$ $\varepsilon(N)$, where $\varepsilon(N)$ is the stochastic error associated with the microscale model. The variance of the stochastic error at any system size $N$ can be given as $\sigma^{2}(N)=$ $\operatorname{Var}(\varepsilon(N))$, calculated by obtaining the variance of $\left\{g_{i}(x, N)\right\}_{i=1 \ldots k}$ for $k$ microscale simulations around the same initial condition. As $N$ increases, less variance is observed around $E[\varepsilon(N)]$. An optimal difference interval $h(N)$ can then be evaluated for any nonlinear programming method as $h(N)=c \sigma^{1 / 3}$ (Brekelmans et al., 2003). The interval scaling factor $c$ is a problem-dependent parameter that is to be chosen by the user based upon a preliminary analysis of the microscale model. After obtaining $h(N)$, this information can then be assimilated into any gradient-based optimization algorithm to obtain accurate derivative information. The idea behind our approach is to increase $N$ as the system optimum is approached. In doing so, the value of $h(N)$ decreases, resulting in increased gradient accuracy around the current point since the length of the difference interval is smaller. The optimum found is expected to be closer to the true minimum than the value obtained using an algorithm employing the same initial value of $N$ throughout. The strategy of this adaptive algorithm relies on limiting the initial microscale information in moving towards the optimum, thereby decreasing the computational overhead involved. Only once the vicinity of the optimum has been reached will the computational expense be increased in an effort to increase the accuracy of the solution. Termination of the algorithm occurs when the norm of the previous and current values of $g(x, N)$, as well as the norm of the previous and current 
solution vector $x$, falls within the expected standard deviation $\varepsilon$ for two successive iterations. The procedure developed is summarized in the flowcharts of Figures 1 and 2.

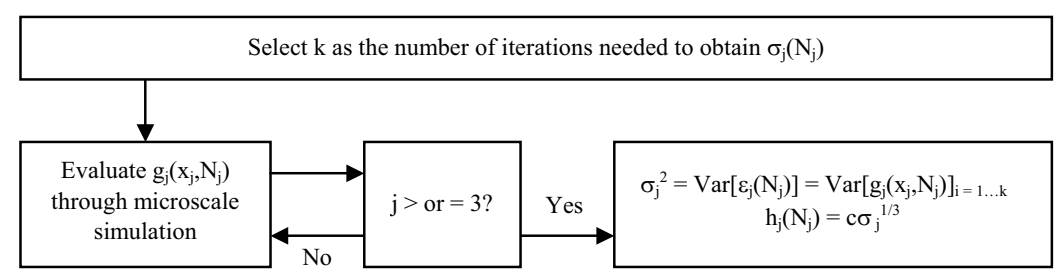

Figure 1: Preliminary analysis to obtain the system noise for the number of particles $\mathrm{N}$

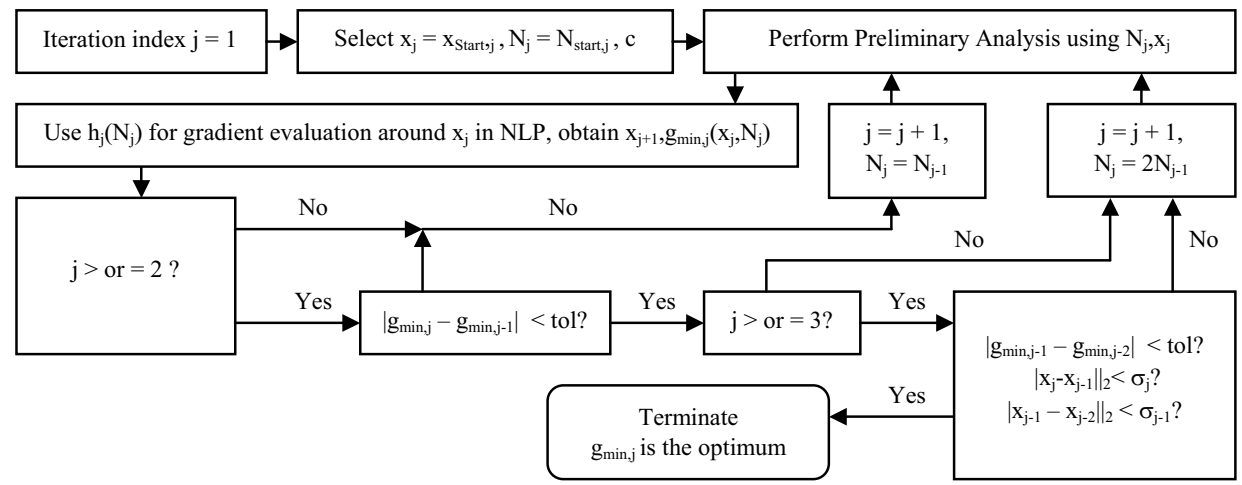

Figure 2: Proposed Gradient-Based Adaptive Algorithm

\subsection{Localized Response Surface Method}

The main idea of response surface methods (RSM) is to first approximate input-output relationships using polynomial functions that can then be optimized via conventional techniques. This surface minimum typically serves as a starting point for subsequent optimization iterations. The approximation can be made over the entire domain or a local region. We use the latter in our approach, which is subsequently moved and/or expanded/contracted depending upon the performance of each iteration. A random point in macroscale space is selected as the center of a hyper-rectangle defined via prespecified bounds. This point is lifted to the microscale level, the system is simulated, and the output vector restricted back to the macroscopic conditions. The response surface is then built over a collocation set of the macroscale input-output vectors, minimized to obtain $P_{\min , j}$, and the system simulated again to obtain $g_{\text {test }, j}\left(\operatorname{argmin} P_{\min , j}\right)$. The next point is obtained at the system minimum of the local region. The algorithm terminates if $\left|g_{\min , j}-g_{\min , j-1}\right|<t o l$, where the $t o l$ is a function of the noise present in the microscale simulation. The polynomial used is quadratic and thus optimality information can be obtained analytically, reducing the computational complexity. Any critical points found outside the feasible region defined by the lower and upper limit bounds on $x$ are discarded. If the criterion is not met, the bounds for the new iteration are then adjusted as described in Figure 3. During the initial iterations of the algorithm, $x_{\min , j}$ will typically be found at the edges of the hyper-rectangle defined by the bounds of the optimization variables. Once the vicinity of the minima is reached, the optimum then 
falls inside the current optimization region. At this point, faster convergence can be achieved by defining the bounds to be the distance between $x_{\min , j}$ and $x_{\min , j-1}$.

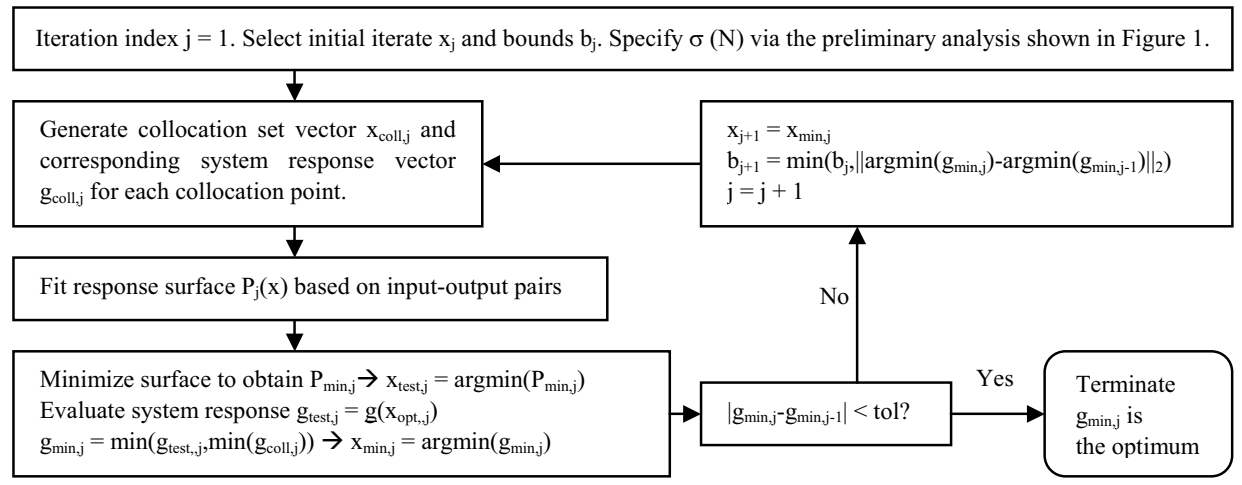

Figure 3. Proposed Localized Response Surface Method

\section{Case Study and Results}

A simple reaction system is selected in order to investigate the effectiveness of the proposed optimization strategies. The reactions are assumed to occur in an ideal CSTR and the reaction network considered is a modification of the Fuguitt and Hawkins mechanism (Floudas and Pardalos, 1995) given by: $\mathrm{A} \rightarrow \mathrm{E}, \mathrm{A} \rightarrow \mathrm{D}, \mathrm{B} \leftrightarrow \mathrm{D}, \mathrm{C} \leftrightarrow 2 \mathrm{D}$, and $2 \mathrm{~A} \rightarrow \mathrm{C}$, where the rate constants are $k_{1}^{f}=3.33384 \mathrm{~s}^{-1}, k_{2}^{f}=0.26687 \mathrm{~s}^{-1}, k_{3}^{f}=$ $0.29425 \mathrm{~s}^{-1}, k_{3}^{r}=0.14940 \mathrm{~s}^{-1}, k_{4}^{f}=0.011932 \mathrm{~s}^{-1}, k_{4}^{r}=0.18957 \mathrm{~L} / \mathrm{mol}, k_{5}^{f}=0.009598$ $\mathrm{L} / \mathrm{mol}, \mathrm{V}=100 \mathrm{~L}, \mathrm{~F}=8 \mathrm{~L} / \mathrm{s}$. Only $C_{A}$ and $C_{C}$ enter the reactor; their ranges are $3<C_{A}{ }^{0}$ $[\mathrm{mol} / \mathrm{L}]<30$ and $0.5<C_{C}{ }^{0}[\mathrm{~mol} / \mathrm{L}]<9.5$. The dynamic behavior of the system is:

$\frac{d C_{A}}{d t}=\frac{F}{V} \cdot\left(C_{A}^{0}-C_{A}\right)-k_{1}^{f} \cdot C_{A}-k_{2}^{f} \cdot C_{A}-k_{5}^{f} \cdot C_{A}^{2}$

$\frac{d C_{B}}{d t}=\frac{F}{V} \cdot\left(-C_{B}\right)-k_{3}^{f} \cdot C_{B}+k_{3}^{r} \cdot C_{D}$

$\frac{d C_{C}}{d t}=\frac{F}{V} \cdot\left(C_{C}^{0}-C_{C}\right)-k_{4}^{f} \cdot C_{C}+0.5 \cdot k_{4}^{r} \cdot C_{D}^{2}+0.5 \cdot k_{5}^{f} \cdot C_{A}^{2}$

$\frac{d C_{D}}{d t}=\frac{F}{V} \cdot\left(-C_{D}\right)+k_{2}^{f} \cdot C_{A}+k_{3}^{f} \cdot C_{B}-k_{3}^{r} \cdot C_{D}+2 \cdot k_{4}^{f} \cdot C_{C}-k_{4}^{r} \cdot C_{D}^{2}$

$\frac{d C_{E}}{d t}=\frac{F}{V} \cdot\left(-C_{E}\right)+k_{l}^{f} \cdot C_{A}$

The objective function is defined as:

$g(X, Y)=4 \cdot(X-0.6)^{2}+4 \cdot(Y-0.4)^{2}+\sin ^{3}(\pi \cdot X)+0.4$

$X=0.1428 \cdot C_{C}^{S S}-0.357 \cdot C_{D}^{S S}$

$Y=-0.1428 \cdot C_{C}^{S S}+2.857 \cdot C_{D}^{S S}+1.0$ 
where $C_{C}^{S S}$ and $C_{D}^{S S}$ represent the macroscale steady-state values of $C_{C}$ and $C_{D}$, respectively. The deterministic solution yields a global optima of $g=0.7422$ at $\left[\mathrm{C}_{\mathrm{A}}{ }^{0}, \mathrm{C}_{\mathrm{C}}{ }^{0}\right]=[10.117,8.378]$ and a local optima of $g=1.2364$ at $[13.202,3.163]$. Steadystate solution vectors are obtained from an initial point by running the microscale simulations for a long time horizon, after which the objective function can be evaluated. The variance of the microscale system error is evaluated to be

$\sigma^{2}=\operatorname{Var}(\varepsilon)=\operatorname{Var}\left(\sum_{i=1}^{k} g_{i}(x, N)\right)$

Sequential quadratic programming (NPSOL) is used to generate the solution of the optimization problem. In the standard mode of optimization, the system size $N$ is fixed for the duration of the algorithm and the difference interval is set to be $h=0.05$. Optimization is then performed using our proposed adaptive algorithm with $c=0.2$ (Figure 2) such that $N$ is increased depending upon the convergence performance at the current iteration of the NLP algorithm. In this method, the difference interval is defined as $h(N)=c \sigma^{1 / 3}$. Using random initial solution vectors, the performance of the adaptive algorithm with respect to the computational time and accuracy of the standard algorithm is reported in Table 1. The "standard" mode of solution refers to a fixed system size, in contrast to the "adaptive" mode referring to a variable system size.

Table 1.Results for the gradient based NLP algorithm for solutions converging to the global solution $\left(C_{A}{ }^{0}, C_{C}{ }^{0}\right)^{\text {opt }}=(10.117,8.378)$, $F=0.7422$

\begin{tabular}{ccccccc}
\hline $\begin{array}{l}\text { Mode of } \\
\text { Solution }\end{array}$ & $\begin{array}{c}\# \text { of } \\
\text { particles N }\end{array}$ & $\left(\mathrm{C}_{\mathrm{A}}{ }^{0}, \mathrm{C}_{\mathrm{C}}{ }^{0}\right)^{\mathrm{opt}}$ & $\begin{array}{c}\text { Std. Dev. } \\
\left(\mathrm{C}_{\mathrm{A}}{ }^{0}, \mathrm{C}_{\mathrm{C}}{ }^{0}\right)^{\text {opt }}\end{array}$ & $\mathrm{F}_{\text {Min }}$ & $\begin{array}{c}\text { Comp } \\
\text { time (s) }\end{array}$ & $\begin{array}{c}\# \\
\text { Func. } \\
\text { Calls }\end{array}$ \\
\hline Standard & $10^{6}$ & $10.167,8.426$ & $0.20,0.05$ & 0.7425 & 2346 & 62 \\
\hline Adaptive & $10^{4}-10^{6}$ & $10.166,8.475$ & $0.21,0.04$ & 0.7440 & 1052 & 96 \\
\hline
\end{tabular}

As can be observed from Table 1, use of the adaptive algorithm results in a substantial reduction in CPU time compared to a standard SQP method, yet requires a larger number of function calls. The value of $N$ is initially lower for the adaptive algorithm, so more iterations are required before the optimum region is reached since reduced models are being used. The standard deviation of the solution for ten runs is comparable.

This optimization problem is also solved using our localized RSM algorithm with ten percent of the domain around $x_{m i n, j}$ set as the optimization region. In each iteration, the collocation set is generated by using a simplex around $x_{m i n, j}$ and the tol is chosen to be proportional to $\sigma$ (in these trials it is the value of $\sigma$ itself), determined as explained above in Fig. 3. The values of the optimal vector $\left[\mathrm{C}_{\mathrm{A}}{ }^{0}, \mathrm{C}_{\mathrm{C}}{ }^{0}\right]^{\text {opt }}$, along with the predicted optima $P_{\text {min }}$, are averaged and reported with the corresponding standard deviation in Table 2, for twenty-five runs each of varying microscale system size. With an increase in the number of particles, the quality of both the solution vector and predicted optimum increases at the expense of the number of function calls. It should be noticed that the user-specified tolerance is found to be an important parameter since relaxing this value increases the chances of the algorithm prematurely terminating at a point away from the 
true optima. At the same time, imposing a very strict criterion leads to the algorithm looping repeatedly with very little improvement in the response surface, even though it may be in a desirable vicinity of the optima.

Table 2: Results for the localized RSM algorithm for solutions converging to the global solution $\left(C_{A}{ }^{0}, C_{C}{ }^{0}\right)^{\text {opt }}=(10.117,8.378), F=0.7422$

\begin{tabular}{ccccccccc}
\hline $\begin{array}{c}\# \\
\text { Particles }\end{array}$ & $\begin{array}{c}\# \\
\text { Copies }\end{array}$ & $\begin{array}{c}\mathrm{N}= \\
\mathrm{Px} \mathrm{C}\end{array}$ & $\sigma$ & $\left(\mathrm{C}_{\mathrm{A}}{ }^{0}, \mathrm{C}_{\mathrm{C}}{ }^{0}\right)^{\text {opt }}$ & $\begin{array}{c}\text { Std. Dev. } \\
\left(\mathrm{C}_{\mathrm{A}}{ }^{0}, \mathrm{C}_{\mathrm{C}}{ }^{0}\right)^{\text {opt }}\end{array}$ & $\mathrm{P}_{\min }$ & $\begin{array}{c}\text { Std. } \\
\text { Dev. } \\
\mathrm{P}_{\min }\end{array}$ & $\begin{array}{c}\text { Func. } \\
\text { Calls }\end{array}$ \\
\hline 10,000 & 1 & 10,000 & 0.0175 & $11.792,8.35$ & $2.044,0.277$ & 0.7172 & 0.0312 & 19 \\
\hline 10,000 & 5 & 50,000 & 0.013 & $9.770,8.060$ & $0.863,0.314$ & 0.7238 & 0.0427 & 27 \\
\hline 100,000 & 1 & 100,000 & 0.011 & $9.962,8.366$ & $0.606,0.041$ & 0.7410 & 0.0050 & 29 \\
\hline 100,000 & 5 & 500,000 & 0.007 & $10.180,8.382$ & $0.186,0.018$ & 0.7423 & 0.0003 & 31 \\
\hline
\end{tabular}

\section{Conclusions and Future Work}

In this work the application of optimization methods using microscopic models is presented. A modified reduced gradient method is developed using microscale system parameters to generate an optimal measure of the difference interval. It is found that the proposed approach leads to a substantial reduction in terms of computational cost compared to standard SQP methods. Moreover, an adaptive RSM method is introduced to take advantage of localized optima information. It is found that a tradeoff exists between the quality of the solution and the number of function calls required. The next step is to integrate these two methodologies, expected to result in a more efficient local optimization approach for noisy functions. Additional efficiency is expected through parallelization of the computations by assigning one microscopic simulation to each CPU. This work is expected to serve as a cornerstone towards developing a global optimization framework for the same class of noisy functions.

\section{References}

Bindal, A., M.G. Ierapetritou, S. Balakrishnan, A. Makeev, I. Kevrekidis and A. Armaou, Submitted for publication, Chem. Eng. Sci., 2004.

Brekelmans R., L. Driessen, H. Hamers, D. den Hertog, 2003, CentER Discussion Paper, ISSN 0924-7815.

Choi, T. D. and C.T. Kelley, 2000, SIAM Journal of Optimization 10,4,1149.

Floudas, C.A. and P.M. Pardalos, 1990, A Collection of Test Problems for Constrained Global Optimization Algorithms.

Gear, C.W., Kevrekidis, I.G., and C. Theodoropoulos, 2002, Comp. \& Chem. Eng., 26, 941.

Jones, D., 2001, J. of Global Optimization, 21, 345.

Jones, D., M. Schonlau, and W. Welch, 1998, J. of Global Optimization, 13, 455.

Kelley, C.T., 1999, Iterative Methods for Optimization, Volume 2, SIAM.

Storn, R. and Price, K., 1995, Differential Evolution - A Simple And Efficient Adaptive Scheme For Global Optimization Over Continuous Spaces, Technical Report, International Computer Science Institute.

\section{Acknowledgements}

The authors gratefully acknowledge financial support from the National Science Foundation under the NSF CTS-0224745 grant. 